\title{
Fauna edáfica em solo construído, campo nativo e lavoura anual
}

\author{
Edafic fauna in constructed soil, native pasture and annual handling farm
}

\author{
Alessandro Samuel Rosa ${ }^{\mathrm{I}}$ Ricardo Simão Diniz Dalmolin ${ }^{\mathrm{II}}$
}

\begin{abstract}
A fauna edáfica é usada para o monitoramento
ade de sistemas agrícolas; em aterros encerrados de RESUMO da qualidade de sistemas agrícolas; em aterros encerrados de
resíduos sólidos urbanos (RSU), o seu estudo, especialmente no Brasil, ainda é incipiente. Foi conduzido um estudo da fauna edáfica do solo construído (SC) sobre um aterro encerrado, em Santa Maria - RS, e em outras duas áreas, sob diferentes usos: campo nativo (CN) e lavoura anual (LA) com manejo ecológico, com vistas a avaliar a qualidade ambiental e a estabilidade desses sistemas. Foram duas coletas, realizadas em janeiro e fevereiro de 2007, tendo sido instaladas, aleatoriamente, oito armadilhas do tipo PROVID, em cada área, por três dias. Houve um predomínio de colêmbolos, himenópteros, ácaros e coleópteros na composição da fauna edáfica dos ambientes estudados. O SC teve a maior abundância de indivíduos, com dominância da Classe Collembola (17,6 vezes maior que em LA), assim como no CN. Na LA, a Ordem Hymenoptera foi a de maior expressão. As alterações meteorológicas durante as épocas de coleta, influenciaram o comportamento dos atributos da fauna edáfica, especialmente em SC e CN. Já em LA foram as alterações na vegetação que determinaram o comportamento da fauna edáfica. A seqüência $L A>C N>S C$ foi a mais representativa para ilustrar as condições qualitativas dos ambientes estudados.
\end{abstract}

Palavras-chave: fauna do solo, aterro encerrado, qualidade do solo, estabilidade ambiental.

\section{ABSTRACT}

Edafic fauna has been used as a biological parameter for monitoring the quality of agricultural systems; in closed landfill sites, its study, especially in Brazil, still is incipient. In that sense, it was carried out a study in a constructed soil (SC) in a closed landfill site at Santa Maria, RS, Brazil taking as comparative two areas under different uses on the neighborhood: native pasture (CN) and an ecologically- managed farm (LA), with the aim to analyze the quality and stability of those systems. Collection was carried out two times in January and February of 2007, when eight PROVID traps were installed aleatorialy in each area during three days. It was observed a prevalence of Collembola, Hymenoptera, Acarina and Coleoptera groups in the composition of the edafic fauna of the studied sites. SC had the largest total abundance, with prevalence of Collembola group (17.6 times greater than in LA), as well as in CN. In LA the Order Hymenoptera was the one of larger expression. Collection times influenced the behavior of edafic fauna attributes in SC and CN, except in LA, where alterations in vegetable community determined its behavior. Sequence $L A>C N>S C$ is the one that best represents the qualitative conditions of the evaluated sites.

Key words: soil fauna, closed landfill, soil quality, environmental stability.

Aterros controlados caracterizam-se pela deposição dos resíduos sólidos urbanos (RSU) sobre solo não impermeabilizado, recebendo cobertura periódica de material, com coleta ou não dos líquidos e gases gerados (MUÑOZ, 2002). Quando atingem sua capacidade máxima, medidas recuperadoras devem ser adotadas, seguindo determinações específicas dos projetos técnicos dos aterros. Nessa fase, os RSU, devem receber uma cobertura de solo que os isole do ambiente adjacente, dando origem ao que se chama solo construído (SC), o qual deve proporcionar o crescimento de vegetação que forme uma comunidade semelhante àquelas de áreas inalteradas e em equilíbrio com os outros componentes ambientais (DWYER, 2000).

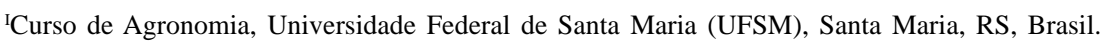

"Departamento de Solos, UFSM, 97105-900, Santa Maria, RS, Brasl. E-mail: dalmolin@pesquisador.cnpq.br. Autor para correspondência. 
A implementação de uma política de monitoramento da qualidade ambiental sobre os SC, após o encerramento dos aterros, é de fundamental importância, podendo ser usados parâmetros como produção de gases (CHAN \& PARKING, 2000) e de lixiviado (OLIVEIRA, 2001), além de caracteres biológicos como a fauna edáfica (CHAN et al., 1997). Esta tem sido usada também no monitoramento da qualidade de sistemas agrícolas (BARETTA et al., 2003; ROVEDDER et al., 2004). Em aterros encerrados de RSU, o uso da fauna edáfica possui um grande potencial como forma de monitorar a evolução destes ambientes.

Neste estudo o objetivo foi avaliar a fauna edáfica em SC sobre aterro encerrado de RSU, campo nativo (CN) e lavoura anual (LA), em duas épocas distintas, com vistas a determinar a qualidade e a estabilidade desses ambientes.

A área de estudo localiza-se no Aterro Controlado da Caturrita (ACC) e adjacências, no Município de Santa Maria-RS, com coordenadas 29 $43^{\prime} 06^{\prime \prime}$ de latitude e $-53^{\circ} 43^{\prime} 45^{\prime \prime}$ de longitude como referência. $\mathrm{O}$ aterro encerrado possui SC resultante da mistura de horizontes A, B e C dos solos do local (Argissolos), com baixos teores de Ca, Mg, K e matéria orgânica (MO), bem como textura franco argilo-arenosa, com predomínio de vegetação espontânea, de porte rasteiro e herbáceo. Aárea de campo nativo $(\mathrm{CN})$ possui vegetação formada por poáceas de porte rasteiro e herbáceo, e solo de baixa fertilidade e reduzida capacidade de retenção de água. A lavoura anual (LA), utilizada há sete anos para o cultivo de plantas anuais no sistema agroecológico, apresenta teores mais elevados de Ca, Mg, P e MO, resultando em maior $\mathrm{pH}$, menor Al trocável e baixa densidade, cultivada, por ocasião das amostragens, com Zea mays L. e Canavalia ensiformis DC., com presença de Brachiaria plantaginea e Richardia brasiliensis.

Para as coletas (C1 e C2 - janeiro e fevereiro de 2007), foram instaladas aleatoriamente oito armadilhas do tipo PROVID (ANTONIOLLI et al., 2006) em cada área, sendo, após três dias, recolhidas e levadas ao laboratório, onde os grupos foram quantificados e identificados quanto à Ordem (GALLO et al., 2002), exceto para colêmbolos e diplópodes, classificados em nível de Classe. Foram calculadas a abundância total, a frequiência relativa e a riqueza e os índices de diversidade de Shannon, de eqüitabilidade de Pielou e de dominância de Simpson (Is), conforme ODUM (1988). A análise estatística foi realizada pelo teste não-paramétrico de Wilcoxon (CAMPOS, 1979), e a abundância de grupos foi avaliada, ainda, pela análise multivariada de agrupamento por meio da distância euclidiana média (JOHNSON \& WICHERN, 1998).
Os grupos capturados no SC apresentaram, em C1, a maior abundância total, com predomínio da Classe Collembola (Tabela 1) cuja abundância foi respectivamente, 1,65 e 17,6 vezes maior que em $\mathrm{CN}$ e LA. Ordens como Acarina, Araneae, Lepidoptera e Thysanoptera também contribuíram para a maior abundância em SC, principalmente, quando comparadas com LA. Em C2, esses grupos continuaram imprimindo diferenças na abundância entre SC em relação a CN e LA, porém com menor intensidade, especialmente a Classe Collembola, igualando-se ao CN. Essa alteração foi provocada pelas condições meteorológicas do período, que foi marcado pela ocorrência de estiagem e grande amplitude térmica. Isso proporcionou o aumento da temperatura e a perda de umidade do solo nesses dois ambientes, o que pode ser observado na vegetação do SC. Como na LA havia uma comunidade de plantas com um dossel de mais de um metro e meio de altura, houve a formação de um microclima, resultando em pequena variação da temperatura e umidade do solo. Isso é importante sob o ponto de vista de que colêmbolos são bastante sensíveis à dessecação, quando submetidos a condições de estresse hídrico (DIDDEN, 1993), situação que também afeta a comunidade fúngica do solo, principal fonte de alimento dessa Classe (HÅGVAR, 1998).

A LA apresentou a maior abundância da Ordem Coleoptera, quando comparada com os demais tratamentos; mas dentro de LA, a Ordem Hymenoptera apresentou a maior abundância. Esses resultados se devem ao grande número de espécies pertencentes a essas Ordens que tem nas culturas agrícolas a sua principal fonte de alimento (GALLO et al., 2002). Quanto aos demais grupos, a abundância encontrada foi menor do que em SC e/ou CN (com exceção de alguns grupos) devido ao pequeno volume de material orgânico na superfície do solo que poderia ser utilizado como alimento (CORREIA \& OLIVEIRA, 2000).

Quanto à freqüência relativa de cada grupo, dentro das comunidades, Collembola, Hymenoptera e Acarina foram os que tiveram maior importância, tanto no SC como no CN, em C1 e C2. Esses grupos ocorrem com maior freqüência em ambientes de $\mathrm{CN}$, conforme constatado por BARETTA et al. (2003) e ROVEDDER et al. (2004). Em SC, sobre aterros sanitários, CHAN et al. (1997) encontraram maior freqüência de Collembola e Acarina. Já na LA, as maiores freqüências foram dos grupos Hymenoptera, Coleoptera, Collembola e Acarina, resultados que vem ao encontro dos obtidos por QUADROS (2004).

As alterações mais significativas ocorridas nos atributos das comunidades avaliadas entre C1 e C2 foram a elevação dos índices de Shannon e de Pielou 
Tabela 1 - Abundância, riqueza, e índices de diversidade de Shannon, de eqüitabilidade de Pielou e de dominância de Simpson da fauna edáfica em solo construído (SC), lavoura anual (LA) e campo nativo (CN) em dois períodos de coleta (C1 e C2). Santa Maria, RS, 2007.

\begin{tabular}{|c|c|c|c|c|c|c|}
\hline \multirow{2}{*}{ Grupos capturados } & \multicolumn{3}{|c|}{--------------------'Primeira coleta----------------------- } & \multicolumn{3}{|c|}{-------------------------Segunda coleta---------------------- } \\
\hline & SC & LA & $\mathrm{CN}$ & SC & LA & $\mathrm{CN}$ \\
\hline Acarina & $54 \mathrm{a} \mathrm{A}^{1}$ & $26 \mathrm{bA}$ & $44 \mathrm{aA}$ & $64 \mathrm{aA}$ & 33bA & $33 \mathrm{bA}$ \\
\hline Araneae & $6 a A$ & $2 \mathrm{bA}$ & $3 \mathrm{bA}$ & $6 a A$ & 2bA & $5 a A$ \\
\hline Phalangida & - & $2 \mathrm{aA}$ & $1 \mathrm{a}$ & $1 \mathrm{a}$ & $2 \mathrm{aA}$ & - \\
\hline Collembola & $440 \mathrm{aA}$ & $25 \mathrm{cB}$ & 267bA & $246 \mathrm{aB}$ & $74 \mathrm{bA}$ & $175 \mathrm{aB}$ \\
\hline Chilopoda ${ }^{2}$ & - & - & - & - & - & - \\
\hline Blattodea $^{2}$ & - & - & - & - & - & - \\
\hline Coleoptera & $25 \mathrm{bA}$ & $46 \mathrm{aA}$ & $6 \mathrm{cA}$ & $8 b B$ & $42 \mathrm{aA}$ & $2 \mathrm{cB}$ \\
\hline Dermaptera $^{2}$ & - & - & - & - & - & - \\
\hline Diptera & $1 \mathrm{aA}$ & ЗаB & 3aA & $2 \mathrm{bA}$ & $12 \mathrm{aA}$ & 3bA \\
\hline Hemiptera & $4 \mathrm{bA}$ & $7 \mathrm{aB}$ & $10 \mathrm{aA}$ & $7 \mathrm{bA}$ & $27 \mathrm{aA}$ & $11 \mathrm{bA}$ \\
\hline Hymenoptera & $59 a A$ & $87 \mathrm{aA}$ & $98 \mathrm{aA}$ & $80 \mathrm{aA}$ & $147 \mathrm{aA}$ & $85 a A$ \\
\hline Lepidoptera & $5 \mathrm{aA}$ & $1 \mathrm{bA}$ & - & $1 \mathrm{aB}$ & $1 \mathrm{aA}$ & - \\
\hline Orthoptera & $2 \mathrm{bA}$ & $6 \mathrm{aA}$ & 4abB & $1 \mathrm{bA}$ & $9 \mathrm{aA}$ & $8 \mathrm{aA}$ \\
\hline Thysanoptera & $9 \mathrm{aA}$ & - & $4 \mathrm{bA}$ & $6 a A$ & $1 b$ & $2 \mathrm{bA}$ \\
\hline Total & 604 & 204 & 439 & 421 & 350 & 323 \\
\hline \multicolumn{7}{|l|}{ Atributos } \\
\hline Riqueza & $10 \mathrm{aA}$ & $9 \mathrm{aA}$ & $9 \mathrm{aA}$ & $10 \mathrm{aA}$ & $11 \mathrm{aA}$ & 8bA \\
\hline Shannon & $0,43 \mathrm{cB}$ & $0,68 \mathrm{aA}$ & $0,50 \mathrm{bB}$ & $0,53 \mathrm{bA}$ & $0,71 \mathrm{aA}$ & $0,54 \mathrm{bA}$ \\
\hline Pielou & $0,43 c B$ & $0,71 \mathrm{aA}$ & $0,52 b B$ & $0,53 \mathrm{bA}$ & 0,70aA & $0,59 \mathrm{bA}$ \\
\hline Simpson & $0,55 \mathrm{aA}$ & $0,28 \mathrm{cA}$ & $0,44 \mathrm{bA}$ & $0,41 \mathrm{aB}$ & $0,26 \mathrm{bA}$ & $0,40 \mathrm{aA}$ \\
\hline
\end{tabular}

1 Médias não seguidas por letras iguais, na linha (minúsculas entre tratamentos e maiúsculas entre coletas), diferem entre si pelo teste de Wilcoxon a 5\% de significância.

${ }^{2}$ Os grupos Chilopoda, Blattodea e Dermaptera alcançaram média inferior a 1 (um).

no SC e no CN, e a redução do índice de Simpson no SC (Tabela 1), especialmente pela redução da abundância do grupo Collembola. Na LA, todos os atributos mantiveram-se estáveis quando comparados C1 e C2; na figura 1, mostra-se, que houve uma grande similaridade entre os grupos capturados na LA, com uma boa distribuição dos extratos, maior até do que no CN. Já em SC, houve a formação de dois extratos distintos: o primeiro com semelhança entre os grupos de mais de $80 \%$ e outro, formado pela Classe Collembola, $100 \%$ diferente dos demais.

O comportamento verificado nos ambientes avaliados pode ser explicado pela análise das características dos solos, das condições meteorológicas do período e da comunidade de plantas de cada ambiente. A análise dos solos evidenciou a maior fertilidade do solo em LA do que em SC e CN, o que favorece o desenvolvimento da comunidade fúngica (FRANSSON et al., 2000) e, portanto, afeta as espécies fungívoras do solo. Contudo, como os ambientes avaliados possuem solos com baixa capacidade de retenção de água, a fauna edáfica foi afetada sobremaneira pela irregularidade de chuvas. Neste caso, a manutenção do solo totalmente coberto em LA favoreceu a fauna edáfica, que se mostrou pouco vulnerável às condições meteorológicas adversas, tendo o seu comportamento condicionado pelas alterações ocorridas na comunidade de plantas. Isso demonstra que alterações periódicas nos atributos do solo e da vegetação influenciam mais a composição da fauna edáfica do que as variações meteorológicas.

Foi observado que a fauna edáfica dos ambientes estudados é predominantemente composta pelos grupos Collembola, Hymenoptera, Acarina e Coleoptera, sendo o primeiro aquele que apresentou a maior dominância no SC e no CN, ambos muito sensíveis às variações meteorológicas do período. Assim, esta Classe serviu para denunciar a fragilidade desses ambientes, dada pelos seus atributos edáficos e composição vegetal, podendo ser utilizada, eficientemente, como um indicador da qualidade ambiental.

É possível afirmar que a seqüência $\mathrm{LA}>\mathrm{CN}>\mathrm{SC}$ (comunidades edáficas) ilustra as

Ciência Rural, v.39, n.3, mai-jun, 2009. 


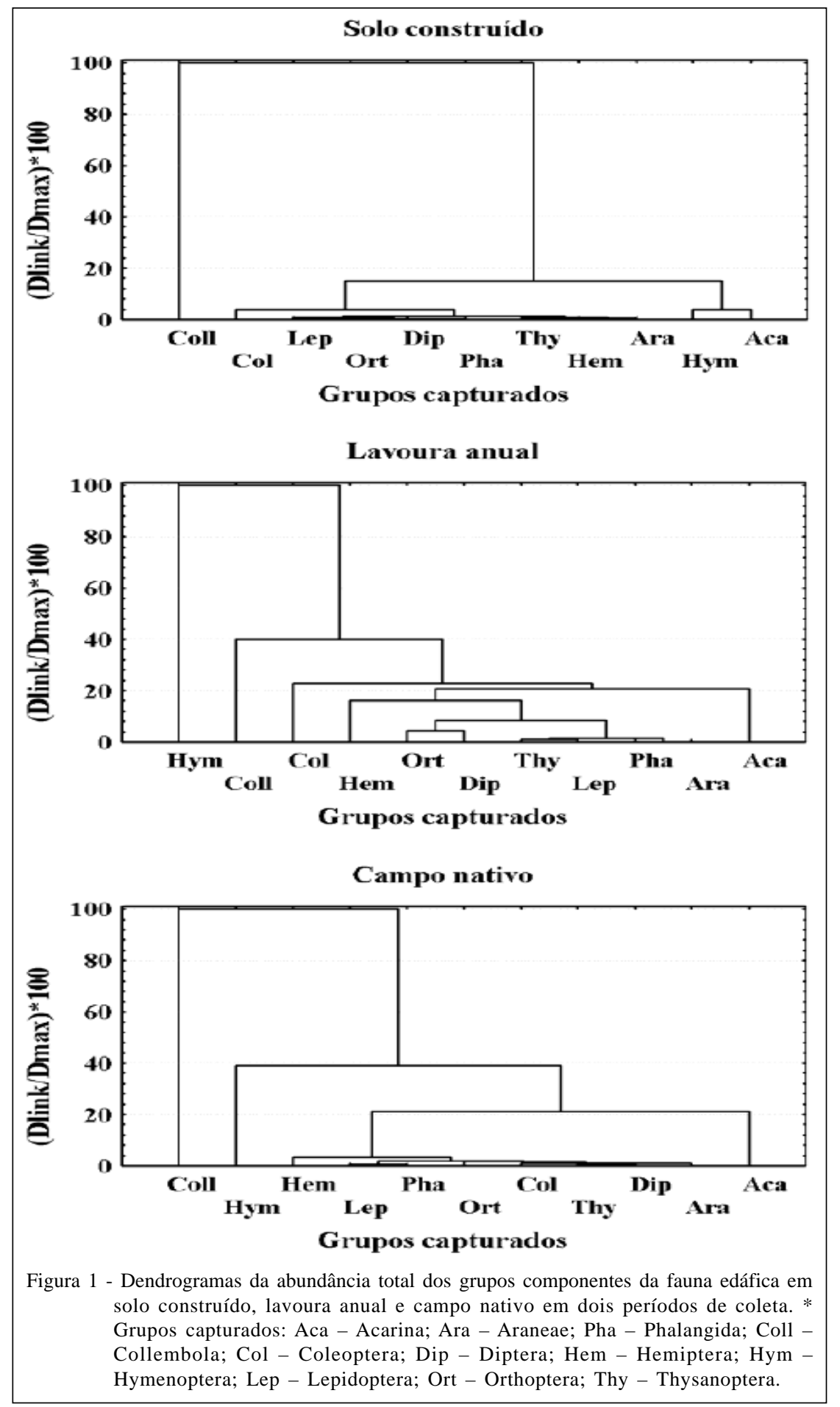

condições qualitativas dos ambientes estudados, diferente do esperado ( $\mathrm{CN}>\mathrm{LA}>\mathrm{SC}$ ), mostrando que as alterações provocadas pela ação antrópica podem produzir melhorias ou retrocessos na qualidade ambiental. Fica evidente a fragilidade da fauna edáfica em áreas de $\mathrm{CN}$, especialmente pela baixa fertilidade e reduzida capacidade de retenção de água dos solos. Neste estudo, mostra-se que, em SC sobre aterros encerrados de RSU, o uso da fauna edáfica como indicador qualitativo deve ser peça fundamental na elaboração de programas de regeneração, já que possui a capacidade de responder a todo e qualquer estímulo ambiental.

Ciência Rural, v.39, n.3, mai-jun, 2009. 


\section{AGRADECIMENTOS}

Ao Conselho Nacional de Desenvolvimento Científico e Tecnológico (CNPQ), pela concessão de bolsa de Produtividade em Pesquisa para Ricardo S. D. Dalmolin e bolsa do Programa Institucional de Bolsas de Iniciação Científica a Alessandro S. Rosa.

\section{REFERÊNCIAS}

ANTONIOLLI, Z.I. et al. Método alternativo para estudar a fauna do solo. Ciência Florestal, Santa Maria, v.16, n.4 p.407-417, 2006.

BARETTA, D. et al. Fauna edáfica avaliada por armadilhas de catação manual afetada pelo manejo do solo na região oeste catarinense. Revista de Ciências Agroveterinárias, v.2, p.97-106, 2003.

CAMPOS, H. de. Estatística experimental não paramétrica. 3.ed. Piracicaba : ESALQ, 1979. 343p.

CHAN, Y.S.G. et al. Influence of landfill factors on plants and soil fauna - an ecological perspective. Environmental Pollution, v.97, n.1-2, p.39-44, 1997.

CHAN, A.S.K.; PARKIN, T.B. Evaluation of potential inhibitors of methanogenesis and methane oxidation in a landfill cover soil. Soil Biology \& Biochemistry, v.32, p.1581-1590, 2000.

CORREIA, M.E.F.; OLIVEIRA, L.C.M. Fauna de solo: aspectos gerais e metodológicos. Seropédica: Embrapa Agrobiologia, 2000. 46p.

DIDDEN, W.A.M. Ecology of terrestrial Enchytraeidae. Pedobiologia, v.37, p.2-29, 1993.

DWYER, S.F. Landfill covers: is it time to change current regulations? Albuquerque: Sandia National Laboratories, 2000. 25p. (Sandia Report).
FRANSSON, P.M.A. et al. Effects of continuous optimal fertilization on belowground ectomycorrhizal community structure in a Norway spruce forest. Tree Physiology, v.20, p.599-606, 2000.

GALLO, D. et al. Manual de entomologia agrícola. Piracicaba: Fealq, 2002. 920p.

HÅGVAR, S. Mites (Acari) developing inside decomposing spruce needles: biology and effect on decomposition rate. Pedobiologia, v.42, p.358-377, 1998.

JOHNSON, R.A.; WICHERN, D.W. Applied multivariate statistical analysis. New Jersey: Prentice Hall, 1998. 816p.

MUÑOZ, S.I.S. Impacto ambiental na área do aterro sanitário e incinerador de resíduos sólidos de Ribeirão Preto, SP: avaliação dos níveis de metais pesados. 2002. 158f. Tese (doutorado em saúde ambiental) - Curso de Pósgraduação de Enfermagem em Saúde Pública, Universidade de São Paulo.

ODUM, E.P. Ecologia. Rio de Janeiro: Guanabara, 1988. 434p.

OLIVEIRA, S. Determinação de alguns parâmetros indicadores de poluição por efluente líquido em um aterro sanitário. 2001. 92f. Tese (Doutorado em Agronomia) - Curso de Pós-graduação em Agronomia, Universidade Estadual Paulista.

QUADROS, V.J. Caracterização biológica nas culturas de batata, soja, feijão e feijão em sistema de cultivo orgânico. 2004. 107f. Dissertação (Mestrado em Ciência do Solo) - Curso de Pós-graduação em Ciência do Solo, Universidade Federal de Santa Maria.

ROVEDDER, A.P. et al. Fauna edáfica em solo suscetível a arenização na região sudoeste do Rio Grande do Sul. Revista de Ciências Agroveterinárias, v.3, n.2, p.87-96, 2004. 